\title{
Underexpression of tumour suppressor LKB1 in clear cell renal cell carcinoma is common and confers growth advantage in vitro and in vivo
}

W C Duivenvoorden ${ }^{1,2,5}$, L K Beatty ${ }^{1,3,5}$, S Lhotak ${ }^{3}$, B Hill ${ }^{3}$, I Mak ${ }^{1,2}$, G Paulin ${ }^{1,2}$, D Gallino ${ }^{1,2}$, S Popovic ${ }^{4}$, R C Austin ${ }^{3}$ and J H Pinthus 1,2,4,* $^{\text {, }}$

${ }^{1}$ Division of Urology, Department of Surgery, McMaster University, Hamilton, Ontario, Canada; ${ }^{2}$ Department of Surgical Oncology, Juravinski Cancer Center, McMaster University, 699 Concession Street, Hamilton, Ontario L8V 5C2, Canada; ${ }^{3}$ Division of Nephrology, Department of Medicine, McMaster University, Hamilton, Ontario, Canada and ${ }^{4}$ Department of Pathology and Molecular Medicine, McMaster University, Hamilton, Ontario, Canada

Background: Evidence suggests that dysregulation of energy-sensing pathways closely associates with renal cell carcinoma (RCC) development. The metabolic regulation is largely controlled by $5^{\prime}$-AMP activated protein kinase (AMPK) which is activated through phosphorylation by LKB1.

Methods: The expression of LKB1 was determined by reverse transcription-PCR using 10 clinical clear cell RCC (ccRCC) samples and their adjacent normal renal parenchyma, and by immunohistochemical staining of two tissue microarrays containing 201 ccRCC and 26 normal kidney samples. Expression of LKB1 was knocked down in human ccRCC 786-O cells (shLKB1) and compared with cells expressing scrambled control shRNA (shControl). AMPK signalling, proliferation, invasion, and VEGF secretion was measured. The cells were subcutaneously injected into mice to determine tumour growth in vivo.

Results: At the protein and transcript levels, a significant reduction in LKB1 expression in tumour compared with normal tissue was found. In vitro, knockdown of LKB1 resulted in reduced AMPK signalling and increased cellular proliferation, invasion, and VEGF secretion compared with shControl cells. In vivo, growth of shLKB1 ccRCC xenografts in nude mice was significantly increased compared with shControl xenografts.

Conclusion: Collectively, our results suggest that LKB1 acts as a tumour suppressor in most sporadic cases of ccRCC and that underexpression of LKB1 is a common event in the disease.

The incidence of renal cell carcinoma (RCC) is steadily rising in Western societies. In the United States, an estimated 58240 new cases and 13040 (22.4\%) deaths occurred in 2010 (Jemal et al, 2010), whereas worldwide these numbers are estimated at 289000 new cases and 102000 (35.3\%) deaths per year (Rini et al, 2009). There are several histological forms of RCC, but the vast majority, $\sim 75 \%$, are clear cell RCC (ccRCC) (Pantuck et al, 2001).
Accumulating evidence suggests that dysregulation of energysensing pathways, a common feature of several hamartoma syndromes, is also critical in the pathogenesis of RCC (Linehan et al, 2010). The metabolic regulation coordinating the cancer cell's energy demands with its energy-consuming malignant phenotype is largely controlled by the $5^{\prime}$-AMP activated protein kinase (AMPK) via the regulation of the mammalian target of rapamycin

\footnotetext{
${ }^{*}$ Correspondence: Dr JH Pinthus; E-mail: jehonathan.pinthus@jcc.hhsc.ca
}

${ }^{5}$ These authors contributed equally to this work.

Received 10 September 2012; revised 26 November 2012; accepted 27 November 2012; published online 15 January 2013 
(mTOR). The AMPK system acts as an 'energy checkpoint' (ratio of cellular AMP/ATP) that determines whether cellular energy status is sufficient before the cell commits to a programme of cell growth triggered by the mTOR pathway. There are also indications that AMPK activation in response to low energy status inhibits cell division by affecting DNA replication and mitosis. Specifically, the catalytic $\alpha$-subunit of AMPK is activated by phosphorylation of Thr172 by one of three upstream kinases: liver kinase B1 (LKB1 or serine/threonine kinase 11 (STK11)), calmodulin-dependent protein kinase kinase- $\beta$, or TGF- $\beta$-activated-kinase 1 (Viollet et al, 2010). The LKB1 tissue-specific knockout mice revealed that LKB1 mediates AMPK activation in the majority of tissues (Shackelford et al, 2009).

Although the major downstream target of LKB1 tumour suppressor is AMPK, LKB1 has at least 12 other substrates, which include members of the AMPK-related protein kinase subfamily, such as MARK1-4 (Lizcano et al, 2004). Activated AMPK turns on catabolic pathways that generate ATP, while inhibiting ATPconsuming processes, such as gluconeogenesis and cell growth. One of the downstream targets of AMPK is the tumour-suppressor protein TSC2 that inhibits mTOR. This has particular significance in RCC (Robb et al, 2007) in which the mTOR pathway is constitutively active. Overall, LKB1-mediated activation of AMPK induces tumour suppression primarily by inhibiting mTOR via activation of TSC2.

The implications of attenuated AMPK activation for tumourigenesis have been demonstrated in other RCC subtypes, including hereditary leiomyomatosis RCC, as a result of inactivation of fumarate hydratase (Tong et al, 2011), in type I papillary RCC through altered HGF/Met signalling, and in patients with BirtHogg-Dubé syndrome who are predisposed to develop chromophobe RCC, as a result of underexpression of folliculin (Linehan et al, 2010). In this study, we hypothesise that attenuated AMPK activation also occurs in ccRCC, the most common form of RCC. We focussed our investigation on LKB1, the key activator of AMPK (Viollet et al, 2010).

\section{MATERIALS AND METHODS}

Human RCC and normal kidney tissue, and cell culture. Frozen specimens of ccRCC and surrounding normal renal parenchyma from 10 patients were used to analyse the expression of LKB1. The study was approved by the institutional Research Ethics Board (protocol no. 02-2174). The histology of the samples was verified to be clear cell carcinoma of the kidney or normal kidney parenchyma by McMaster University's staff pathologists using haematoxylin and eosin (H\&E)-stained sections. Two tissue microarrays (TMAs) were obtained from US Biomax (KD951 and KD6161; Rockville, MD, USA). They contained different renal tissue specimens, but primarily ccRCC (248), and 34 normal kidney specimens.

Mycoplasma-free human ccRCC 786-O cells (ATCC, Manassas, VA, USA) were propagated in RPMI-1640 medium supplemented with 10\% FBS (Invitrogen, Burlington, ON, Canada). The cells' identity was verified by STR analysis (ATCC).

Infection and short-hairpin RNA. The shRNA-mediated knockdown of LKB1 was performed using shRNA lentiviral particles (Santa Cruz Biotechnology, Santa Cruz, CA, USA) designed to knock down LKB1 expression (shLKB1). The shRNA lentiviral particles with a noneffective scrambled shRNA sequence (shControl) were used as negative control. The 786 -O cells $\left(4.0 \times 10^{4}\right.$ cells per well) were seeded into a 12-well plate. The following day, $25 \mu \mathrm{l}$ of shRNA lentiviral particles (in $1 \mathrm{ml}$ supplemented RPMI-1640 media containing $5 \mu \mathrm{g} \mathrm{ml}^{-1}$ polybrene) were added for $24 \mathrm{~h}$. The shLKB1 and shControl cells were selected using $2 \mu \mathrm{g} \mathrm{ml}^{-1}$ puromycin.
Quantitative reverse transcription-PCR. To determine the relative expression of LKB1, RNA isolated from $75 \mathrm{mg}$ freshfrozen human tissue was reverse transcribed using $2.5 \mu \mathrm{g}$ RNA using the SuperScript-VILO cDNA synthesis kit (Invitrogen). For each target gene in triplicate, cDNA was amplified using SYBRGreen PCR master mix (Applied Biosystems; Life Technologies Inc., Burlington, ON, Canada) containing $200 \mathrm{~nm}$ forward and reverse gene primers for human LKB1 (Wang et al, 2012) or $\beta$ actin (Sloan et al, 2009). The PCR was started as $2 \mathrm{~min}$ at $50^{\circ} \mathrm{C}$, $10 \mathrm{~min}$ at $95^{\circ} \mathrm{C}$, followed by 40 cycles of $1 \mathrm{~min}$ at $60^{\circ} \mathrm{C}$, and $15 \mathrm{~s}$ denaturation at $94^{\circ} \mathrm{C}$. The $2^{-\Delta \Delta \mathrm{C} \tau}$ method (Livak and Schmittgen, 2001) was used to calculate relative expression levels.

Western blot analysis. Protein from $75 \mathrm{mg}$ fresh-frozen human tissue was isolated using the Ambion PARIS kit (Life Technologies Inc.). Human ccRCC cells were lysed using RIPA lysis buffer. A total of $40 \mu \mathrm{g}$ lysate was resolved on a $10 \%$ SDS-PAGE gel and transferred onto nitrocellulose membrane. Primary antibodies used were AMPK $\alpha$, phosphorylated(Thr172)-AMPK $\alpha$ and LKB1 ( $1: 1000$, Cell Signaling Technology, Boston, MA, USA), and $\beta$ actin $(1: 2000$, Sigma-Aldrich, Oakville, ON, Canada). Secondary HRP-conjugated antibody ( $1: 200$, Dako, Carpinteria, CA, USA) was used in conjunction with chemiluminescence detection.

VEGF-ELISA assay. The shLKB1 and shControl cells $\left(7.5 \times 10^{4}\right.$ cells per well) were seeded into a 96-well plate in $100 \mu$ l supplemented RPMI-1640 medium and grown overnight. The cells were washed three times with PBS. Following a 24-h incubation in $1 \%$ FBS-supplemented RPMI, the conditioned medium was collected and the amount of VEGF determined using human VEGF ELISA-kit (R\&D Systems, Minneapolis, MN, USA).

Invasion and migration assays. Invasion and migration of shControl and shLKB1 cells $\left(2.5 \times 10^{4}\right.$ cells per well $)$ was measured using 24-well Matrigel invasion chamber kits (BD Biosciences, Bedford, MA, USA) according to the manufacturer's instructions. After $24 \mathrm{~h}$, the invading/migrating cells were stained with crystal violet and counted in five fields of view ( $\times 40$ objective) for each insert. The average number of invaded cells was divided by the average number of migrated cells to determine percentage invasion.

Immunohistochemistry and image analysis. A single formalinfixed, paraffin-embedded block was prepared for each patient's tumour tissue and normal surrounding parenchyma. Immunostaining on $4 \mu \mathrm{m}$-thick sections was performed using heat-induced antigen retrieval (pre-heated $10 \mathrm{~mm}$ citric acid buffer $(\mathrm{pH}=6.0)$ for $30 \mathrm{~min}$ ) and rabbit anti-LKB1 antibody (Abcam, Cambridge, MA, USA) or anti-Ser235/236 phosphorylated-S6 ribosomal protein antibody (Cell Signaling Technology). Controls included murine muscle tissue (positive control) and omission of primary antibody (negative control). The TMAs were immunostained for LKB1 using the same protocol and digitised on AperioScan XT (Aperio Technologies Inc., Vista, CA, USA). The adjusted $H$-score for each core was determined as a measure of staining intensity. By semiautomatic quantification using the positive pixel count (v9) algorithm provided by the ImageScope software (version 11.1.2.760, Aperio Technologies), the staining intensity of each pixel in the TMA cores was determined. Brown pixels, with a hue value of 0.1 and hue width of 0.5 , were considered negative (0), weak positive (1), positive (2), or strong positive (3) using the default intensity threshold parameters from the positive pixel count algorithm. Pixels not meeting the hue limits were ignored. The $H$-score calculation was adapted from Helin et al (1988) as follows: $\Sigma(i+1) \times P i$, in which $i=$ pixel staining intensity (range $0-3)$ and $P i=\%$ of stained pixels. For each individual, the adjusted $H$-score was calculated by subtraction of the $H$-score of the negative control (144.8) and averaging the duplicate $H$-scores. 
Animal studies. All protocols for animal studies were reviewed and approved by the institutional Animal Research Ethics Board. Per group, 10 male inbred nude (Balb/c nu/nu) mice (Charles River, Wilmington, MA, USA), 5 weeks of age, were used. The shLKB1 and shControl cells $\left(2 \times 10^{6}\right.$ in $50 \%(\mathrm{v} / \mathrm{v})$ Matrigel) were injected subcutaneously into the right flank of the mouse. Tumour size was measured weekly using Vernier calipers and tumour volume was determined using the formula $\pi / 6$ (length $\times$ width $\times$ height). The mice were killed 7 weeks after injection, when the tumours were dissected, weighed, fixed in formalin, and embedded in paraffin.
A

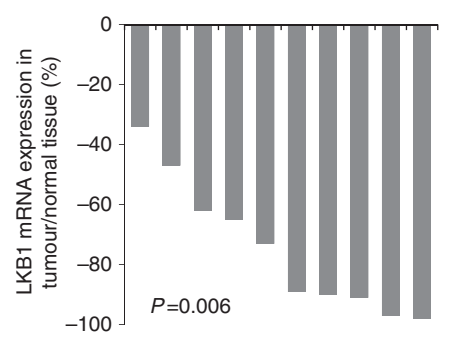

B

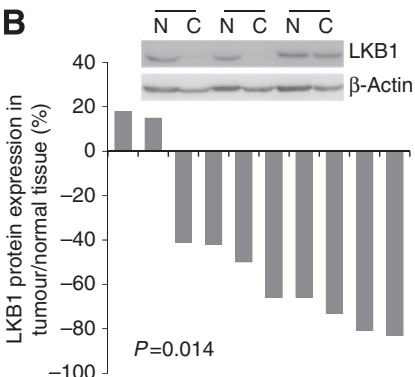

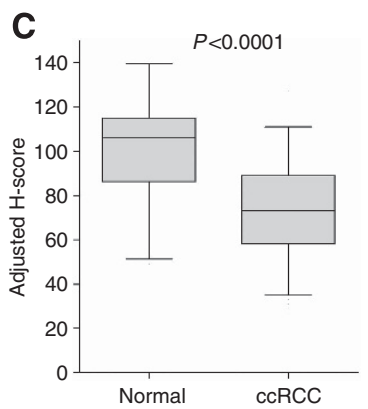

Figure 1. The expression of LKB1 is reduced in human ccRCC tissue. (A) The mRNA expression of LKB1 in 10 out of 10 ccRCC specimens examined was significantly lower than the expression of LKB1 in their adjacent normal parenchyma $(P=0.006)$, as determined by quantitative reverse transcription-PCR analysis. The LKB1 expression in the cancerous tissue is expressed as a percent difference of the expression found in the corresponding adjacent normal tissue (normalised for the expression of actin). At least three different experiments were carried out in triplicate. (B) The protein expression of LKB1 in 8 of 10 specimens of ccRCC studied was significantly lower than the expression of LKB1 in the adjacent normal parenchyma $(P=0.014)$. Protein expression was determined by Western blot analysis and quantified by densitometry (normalised for the expression of actin). The LKB1 protein expression in the cancerous tissue is expressed as a percent difference of the protein expression found in the corresponding adjacent normal tissue. At least three different experiments were carried out in triplicate. A representative Western blot for LKB1 for three patients ( $C=$ cancerous tissue; $N=$ adjacent normal parenchyma) is shown. The two samples with slight increases in LKB1 protein expression correspond to the third and fourth sample from (A) with a $62 \%$ and $63 \%$ decrease in mRNA expression in the tumour vs adjacent normal. (C) Box plots comparing the adjusted $\mathrm{H}$-score ( \pm confidence interval) of LKB1 immunohistochemical staining intensity of normal kidney tissue $(n=26)$ with staining intensity of RCC tissue $(n=201)$. $P<0.0001$ (Student's t-test). The top and bottom quartiles and the median values are depicted as box plot.
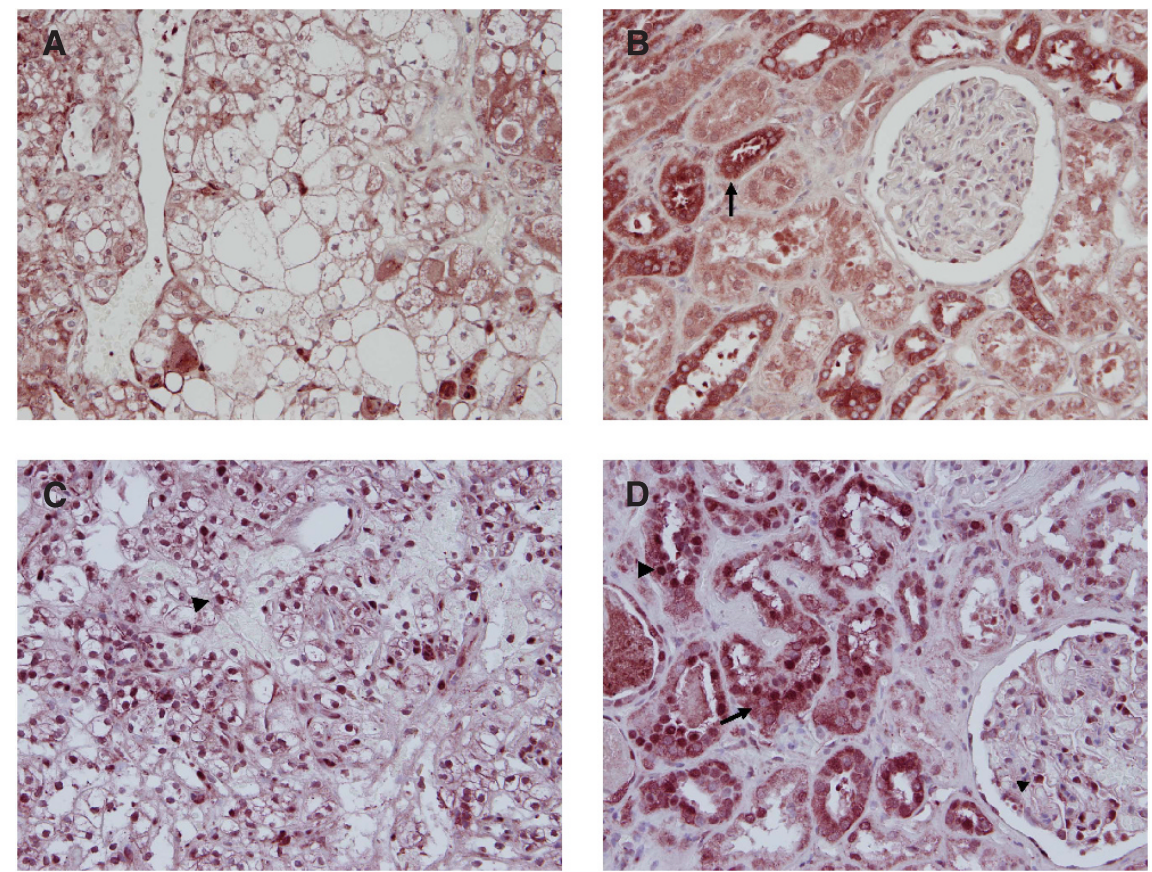

ccRCC

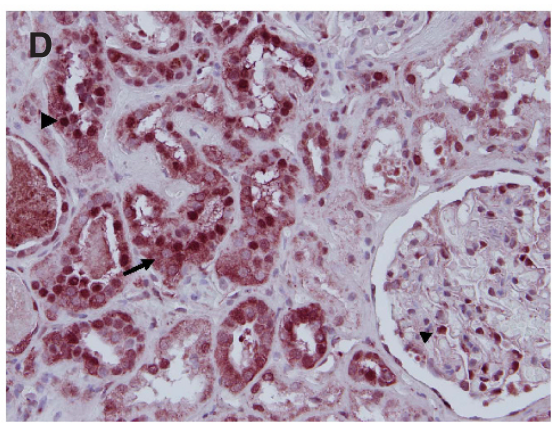

Normal

Figure 2. Immunohistochemistry for LKB1 in human renal and ccRCC tissue. (A) Photomicrographs of LKB1 immunohistochemistry of human cCRCC tissue. (B) LKB1 immunohistochemistry of human normal kidney tissue adjacent to human ccRCC tissue shown in (A). As in most cases of ccRCC, staining for LKB1 was primarily in the cytoplasm of the tubular epithelial cells (arrow). (C) LKB1 immunohistochemistry of another case of human ccRCC. Staining for LKB1 was found primarily in the nuclei of tumour cells (arrowhead). (D) LKB1 immunohistochemistry of normal human renal tissue adjacent to human ccRCC tissue shown in (C). Staining for LKB1 was found in the cytoplasm (arrow), but also in the nuclei of tubular epithelial cells as well as the glomeruli (arrowheads). Original magnifications $\times 400$. 
Statistical analysis. Values are given as the mean \pm s.e.m. Where appropriate, results are presented with 95\% confidence intervals. Dependent on whether the data were normally distributed or not, parametric (Student's $t$-test) or nonparametric methods (MannWhitney $U$-test) were used with a $P$-value of $<0.05$ considered significant.

\section{RESULTS}

Underexpression of LKB1 in clinical specimens of human ccRCC. The expression levels of LKB1 in frozen radical nephrectomy specimens of $10 \mathrm{ccRCC}$ patients were determined by RT-PCR and Western blot analysis. We compared the LKB1 levels in the tumours with those in the normal surrounding renal parenchyma in each of the patients. The expression of LKB1 in all 10 cases of ccRCC examined was significantly lower (mean $74.6 \% \pm 13.7 \%$ reduction) in the tumour samples than in the adjacent normal parenchyma (Figure $1 \mathrm{~A}, P=0.006$, Wilcoxon rank-sum test).

The LKB1 mRNA results were confirmed by protein expression levels in the same clinical samples, where overall LKB1 protein was also significantly decreased (mean $46.9 \% \pm 22.6 \%$ reduction) in the tumour samples compared with adjacent normal tissue (Figure 1B, $P=0.014)$. Immunohistochemistry for LKB1 as shown in Figure 2 also clearly demonstrates that LKB1 expression was decreased in ccRCC tissue compared with normal surrounding renal tissue. In ccRCC tissue (Figure 2A), immunostaining for LKB1 is found in both the nucleus and cytoplasm of the tumour cells. In normal kidney (Figure 2B), the staining is much more prominent in the cytoplasm of the tubular epithelial cells, although the staining is very heterogeneous. Some cases demonstrate much more prominent nuclear LKB1 staining (Figure 2C and D) with nuclear positivity in some glomeruli of normal kidney as well. To further determine the clinical relevance of LKB1 in ccRCC, two TMAs were immunohistochemically stained for LKB1. The two TMAs consisted of normal renal tissue $(n=26)$, and 201 quantifiable primary ccRCC specimens. Consistent with our Western blot analysis on frozen nephrectomy specimens, we also observed a significant decrease in the adjusted $H$-score in the ccRCC samples compared with normal kidney tissue (Figure $1 \mathrm{C}, P<0.0001$ ). By Pearson's correlation analysis, decreased LKB1 staining with increased stage $(P=0.059)$ was observed, although nonsignificantly, likely because of the very small numbers of samples from stage III $(n=21)$ and IV $(n=5)$ disease.

Knockdown of LKB1 expression in human ccRCC cells leads to increased proliferation, VEGF secretion, and invasion in vitro. Western blot analysis of a panel of human and murine kidney-related cell lines (Figure 3A) demonstrates that LKB1 was expressed in each cell line, although to a lesser extent in the murine RCC cell lines. Similar to what was observed in the clinical samples (Figure 1B), LKB1 protein expression in human ccRCC cell lines was also decreased (87.7-99.2\%) when compared with immortalised human embryo kidney HEK293 cells. To recapitulate the clinical observation of decreased LKB1 expression and investigate the consequences of decreased LKB1 in ccRCC, shRNA specific to LKB1 was used to knock down the expression of LKB1 in human ccRCC 786-O cells that, among the human ccRCC cell lines, have intermediate LKB1 expression.

The effects on proliferation, and on two essential steps in the metastatic process, the secretion of VEGF and invasion and
A

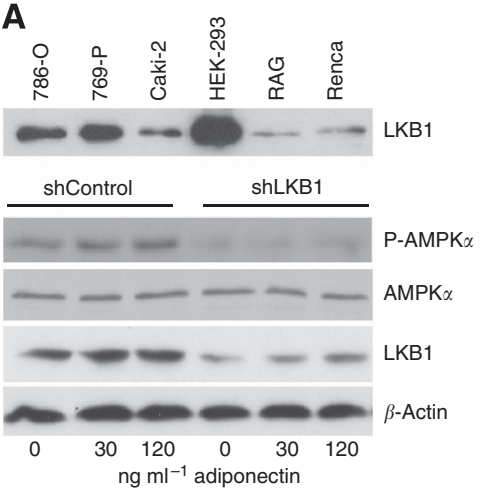

C

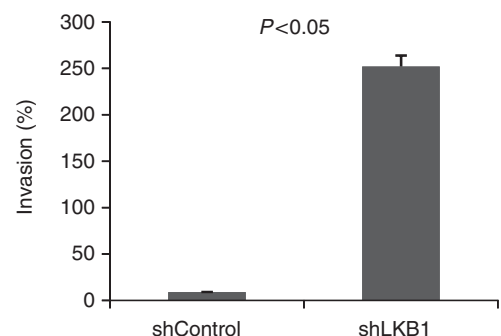

B
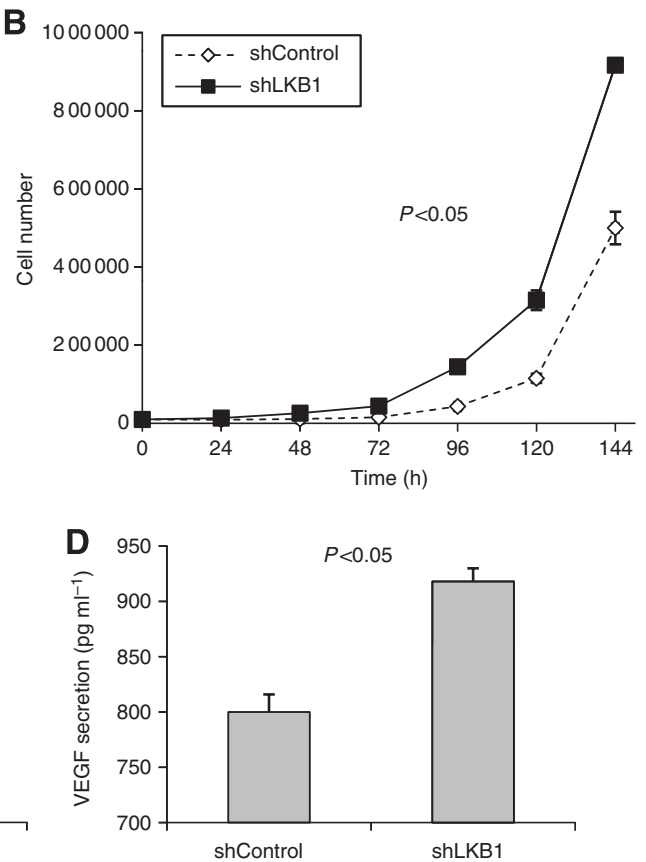

Figure 3. Effect of LKB1 knockdown on AMPK signalling, proliferation, VEGF secretion, and the invasive and migratory potential of RCC cells in vitro. (A) Western blot analysis for LKB1 expression in a panel of human (786-O, 769-P, and Caki-2) and murine (RAG and Renca) RCC, and transformed human embryonic kidney cells (HEK293) (top panel), and in 786-O cells transfected with shRNA specific for LKB1 (shLKB1) or noneffective scrambled shRNA (shControl) (bottom panel). LKB1 protein expression (normalised for actin) is $47 \%$ reduced in the shLKB1 cells compared with shControl cells. Treatment with increasing concentrations of adiponectin, a known activator of AMPK, for $1 \mathrm{~h}$ increases phosphorylation (activation) of AMPK as determined by Western blot analysis, an effect abolished in cells transfected with shLKB1 (bottom panel). (B) The rate of proliferation of the shControl and shLKB1 cell lines was determined by cell counting every day over 6 days. At all time points, the number of shLKB1 cells was significantly larger than the corresponding number of shControl cells ( $t$-test, $P<0.05$ ). (C) Invasion through Matrigel is significantly increased in shLKB1 cells compared with corresponding shControl cells. Invasion is expressed as a percentage of invaded/migrated cells. (D) The shLKB1 cells secreted significantly larger amounts of VEGF than shControl cells. The amount of VEGF secreted into the conditioned medium of shLKB1 or shControl cells over $24 \mathrm{~h}$ was measured using ELISA. Data represent mean \pm s.e.m. 
migration, were determined. Both processes have been shown to associate clinically with metastatic dissemination in RCC patients (Fukata et al, 2005). Human ccRCC 786-O cells stably expressing shRNA specific to LKB1 (shLKB1) demonstrated a $47 \%$ knockdown of LKB1 protein expression compared with 786-O cells stably expressing noneffective scrambled control shRNA (shControl) (Figure 3A). Furthermore, when increasing concentrations of adiponectin, a known activator of AMPK (Lu et al, 2012), were used to treat cells, we demonstrate that the activation of AMPK was virtually abolished in shLKB1 cells when compared with shControl cells. The rate of proliferation was also significantly higher in shLKB1 cells than in shControl cells (Figure 3B). At all time points tested, the number of shLKB1 cells was significantly larger than the corresponding number of shControl cells ( $t$-test, $P<0.05)$. Knockdown of LKB1 in human 786-O cells also led to an almost 30-fold increase in Matrigel invasion when compared with shControl cells (Figure 3C, $t$-test, $P<0.05$ ). Moreover, whereas human shControl cells secreted $800 \pm 27 \mathrm{pg} \mathrm{ml}^{-1}$ VEGF over a 24-h period, the VEGF secretion in shLKB1 cells was increased to $918 \pm 17 \mathrm{pg} \mathrm{ml}^{-1}$ (Figure 3D, $t$-test, $P<0.05$ ).

Knockdown of LKB1 expression in human ccRCC xenografts leads to larger tumours and faster tumour growth in vivo. Mice were subcutaneously injected with human shLKB1 or shControl
ccRCC cells. When human ccRCC 786-O cells were subcutaneously injected into nude mice, not all mice developed tumours. For the shControl cells, the tumour take rate amounted to $50 \%$, whereas this increased to $90 \%$ for mice injected with shLKB1 cells (data not shown). Consistent with our in vitro findings, the tumour volume of subcutaneously growing shLKB1 cells was significantly larger than shControl tumours at all time points tested $(P<0.05$, Mann-Whitney $U$-test $)$, except for weeks 2 and 5 post injection (Figure $4 \mathrm{~A}$ ). The tumour weights of shLKB1 cell-injected mice killed 7 weeks after subcutaneous injection were also significantly higher than those of shControl tumours (Figure 4B, $P=0.016$, Mann-Whitney $U$-test). H\&E staining confirmed the presence of tumour cells in the nodules (Figure $5 \mathrm{~A}$ and B). The LKB1 immunohistochemistry of tumour sections demonstrates that the knockdown of LKB1 remained stable in vivo, as there was clearly less LKB1 staining in the shLKB1-injected mice (Figure 5D) than in the shControl mice (Figure 5C). Similar to human clinical samples, the staining for LKB1 was found in the cytoplasm of most tumour cells. Expectedly, the decrease in LKB1, due to the subsequent decreased activation of AMPK, was associated with an increased activation of S6 ribosomal protein at Ser235/236 as shown by immunohistochemistry in shLKB1 tumours (Figure 5F) compared with the shControl tumours (Figure 5E).
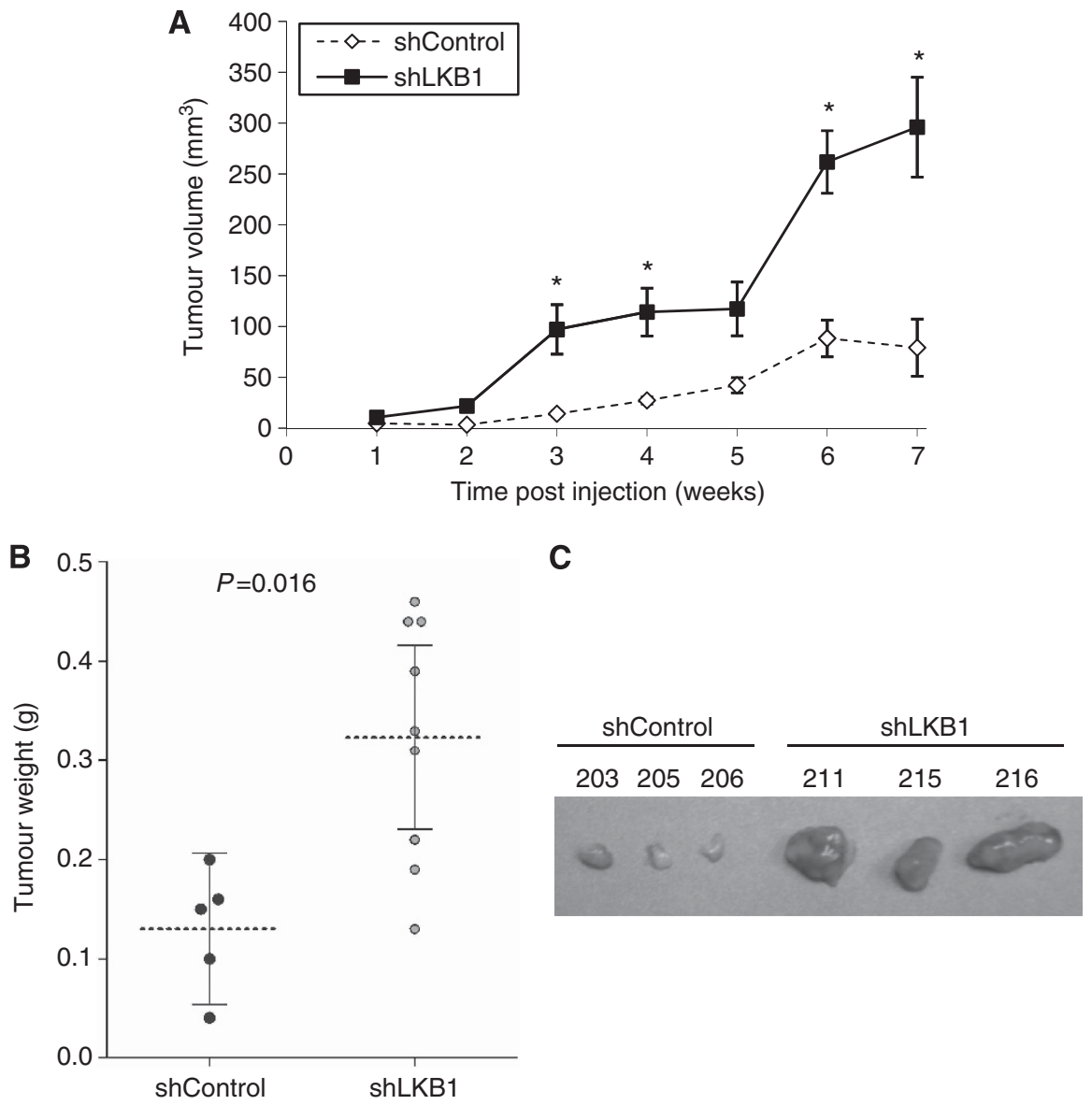

Figure 4. Knockdown of LKB1 expression in human RCC cells results in larger tumours in vivo. (A) Tumour volume ( \pm s.e.m.) from mice injected s.c. with shLKB1 cells $(n=9)$ was significantly higher than from mice injected with shControl cells $(n=5)$. *Statistically significantly different from the volume of the corresponding shControl-injected mice using the Mann-Whitney U-test. (B) Tumour weight of shLKB1-injected mice killed 7 weeks after injection was significantly higher than of shControl-injected mice ( $P=0.016$, Mann-Whitney U-test). Individual values, $95 \%$ confidence interval, and mean (dashed line) are shown. (C) Photomicrographs of three mouse tumours in each group with tumour volume closest to the mean at the time of killing at 7 weeks after injection of the tumour cells. Two out of these three nodules (mouse 205 and 206) in the control group did not contain tumour cells, but only muscle and fat. 

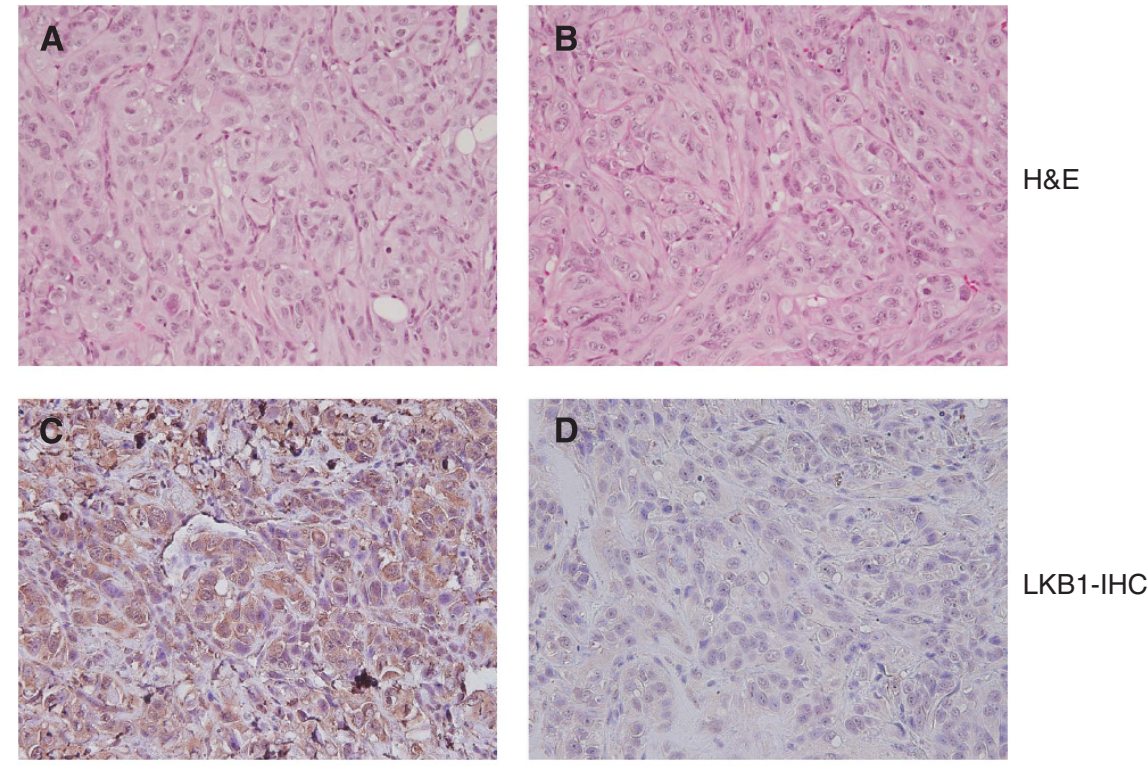

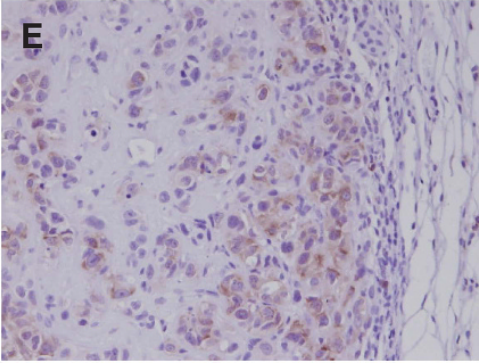

shControl

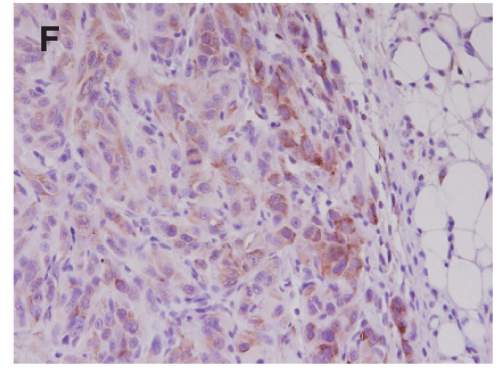

ShLKB1

P-S6-IHC

Figure 5. Haematoxylin and eosin (H\&E) staining and immunohistochemistry for LKB1 and S6 ribosomal protein. Photomicrographs of tumour sections from nude mice injected with shControl or shLKB1 cells stained using H\&E (A and B). Immunohistochemistry clearly demonstrated decreased immunostaining for LKB1 in the shLKB1-derived tumours (D) than the shControl-injected (C) mice. Staining for LKB1 was primarily found in the cytoplasm of the tumour cells. The shLKB1-derived tumours (F) showed more intense immunostaining for phosphorylated (Ser235/236) S6 ribosomal protein than the shControl-injected $(\mathrm{E})$ mice. Original magnifications $\times 400$.

\section{DISCUSSION}

The vast majority of patients with ccRCC overexpress VEGF in tumour tissues, both at the transcript and protein levels. In all our 10 clinical samples examined, expression of VEGF was also increased (data not shown), confirming the validity of our experiments. This increase in VEGF may, at least in part, result from the inactivation of the von Hippel-Lindau (VHL) tumoursuppressor gene that occurs in $60 \%$ of patients. Inactivation of VHL impairs the normal degradation process of hypoxia-inducible factor (HIF)- $1 \alpha$, resulting in transcriptional upregulation of a series of HIF-target genes, including VEGF (Rini and Small, 2005). The shLKB1 cells also demonstrated a significant increase in VEGF secretion (Figure 3D). Thus, in human ccRCC 786-O cells, which are VHL and HIF- $1 \alpha$ deficient (Shinojima et al, 2007), underexpression of LKB1 promotes VEGF secretion, potentially mediated through HIF- $2 \alpha$ (Sowter et al, 2003). The results obtained with 786-O ccRCC cells clearly demonstrate a tumoursuppressive role for LKB1. Future investigations will include the contributions of VHL and HIF transcription factors, for which other RCC cell lines not deficient for these proteins will be employed to further confirm our findings.

From a tumourigenesis point of view, it may have been more straightforward to overexpress LKB1 in ccRCC cells and determine the tumour-suppressive effect. However, as can be seen from
Figure 3A, all RCC cell lines express LKB1, and attempts to induce significant further overexpression of LKB1 were not meaningful. Transfected cells with higher expression levels of LKB1 did express increased activated AMPK and showed reduced proliferation and VEGF secretion (data not shown), but these results did not achieve statistical significance, most likely because the parental cells already express sufficient LKB1. Importantly, our clinical data show that the tumour samples all have substantially reduced LKB1 protein and mRNA expression (Figure 1). For these reasons, we were more interested in pursuing the knockdown of LKB1 in our ccRCC cell lines to recapitulate the clinical observation of decreased LKB1 expression and investigate the consequences of decreased LKB1 in ccRCC. Nevertheless, in support of our novel results in ccRCC cells, in breast and oesophageal cancer, LKB1-overexpressing cells show decreased invasion and migration compared with corresponding control cells (Zhuang et al, 2006; Taliaferro-Smith et al, 2009; Gu et al, 2012).

Truncating germline mutations in $L K B 1$ on chromosome $19 \mathrm{q}$ were found to be responsible for the predisposition of patients with Peutz-Jeghers syndrome (Hemminki et al, 1998), a rare, autosomal dominant disorder characterised by the presence of hamartomous polyps in the gastrointestinal tract. Although these patients are at an increased risk of developing tumours in the gastrointestinal tract, they, interestingly, do not appear at increased risk of RCC, perhaps, in part, because of the rare prevalence of this syndrome and the early age of mortality from it. Our data show for the first 
time that LKB1 is also underexpressed in the majority of ccRCC. Although loss of LKB1 by itself may not be sufficient for RCC initiation, it may be important in progression because of its effects on angiogenesis and invasion as demonstrated here. LKB1 has been reported to be reduced in other sporadic cancers, including in breast cancer (Fenton et al, 2006), biliary and pancreatic (Sahin et al, 2003), and lung carcinomas (Ghaffar et al, 2003). Interestingly, as opposed to these cancer types in which a relatively small proportion of the tumours (2.6-26\%) underexpress LKB1 (Ghaffar et al, 2003; Sahin et al, 2003; Fenton et al, 2006), our data indicate that LKB1 is more universally reduced in ccRCC (Figure 1). Although in 19 renal tumours studied, no somatic mutations of LKB1 were found (Avizienyte et al, 1999), epigenetic changes, such as promoter hypermethylation, can also lead to inactivation of tumour-suppressor genes as has been shown for LKB1 in a small subset of sporadic colorectal tumours (Trojan et al, 2000) and in 5 out of 11 papillary breast carcinomas (Esteller et al, 2000).

Our in vitro and in vivo results also indicate, for the first time, that underexpression of LKB1 may significantly affect ccRCC progression. Moreover, underexpression of LKB1 in ccRCC complements the accumulating understanding that kidney cancer is a disease of cell metabolism with AMPK underactivation as the common molecular hub which was only demonstrated for other less common subtypes of RCC until now (Linehan et al, 2010).

\section{ACKNOWLEDGEMENTS}

We are indebted to Dr A Kapoor (St Joseph's Healthcare, Hamilton, ON, Canada) for providing the 10 renal tumour samples. This work was supported by the Canadian Institutes of Health (Grant 93465 to JHP) and an early career award by Hamilton Health Sciences Corporation (to JHP).

\section{REFERENCES}

Avizienyte E, Loukola A, Roth S, Hemminki A, Tarkkanen M, Salovaara R, Arola J, Butzow R, Husgafvel-Pursiainen K, Kokkola A, Jarvinen H, Aaltonen LA (1999) LKB1 somatic mutations in sporadic tumors. Am J Pathol 154: 677-681.

Esteller M, Avizienyte E, Corn PG, Lothe RA, Baylin SB, Aaltonen LA, Herman JG (2000) Epigenetic inactivation of LKB1 in primary tumors associated with the Peutz-Jeghers syndrome. Oncogene 19: 164-168.

Fenton H, Carlile B, Montgomery EA, Carraway H, Herman J, Sahin F, Su GH, Argani P (2006) LKB1 protein expression in human breast cancer. Appl Immunohistochem Mol Morphol 14: 146-153.

Fukata S, Inoue K, Kamada M, Kawada C, Furihata M, Ohtsuki Y, Shuin T (2005) Levels of angiogenesis and expression of angiogenesis-related genes are prognostic for organ-specific metastasis of renal cell carcinoma. Cancer 103: 931-942.

Ghaffar H, Sahin F, Sanchez-Cepedes M, Su GH, Zahurak M, Sidransky D, Westra WH (2003) LKB1 protein expression in the evolution of glandular neoplasia of the lung. Clin Cancer Res 9: 2998-3003.

Gu Y, Lin S, Li JL, Nakagawa H, Chen Z, Jin B, Tian L, Ucar DA, Shen H, Lu J, Hochwald SN, Kaye FJ, Wu L (2012) Altered LKB1/CREB-regulated transcription co-activator (CRTC) signaling axis promotes esophageal cancer cell migration and invasion. Oncogene 31: 469-479.

Helin HJ, Helle MJ, Helin ML, Isola JJ (1988) Immunocytochemical detection of estrogen and progesterone receptors in 124 human breast cancers. Am J Clin Pathol 90: 137-142.

Hemminki A, Markie D, Tomlinson I, Avizienyte E, Roth S, Loukola A, Bignell G, Warren W, Aminoff M, Hoglund P, Jarvinen H, Kristo P,

Pelin K, Ridanpaa M, Salovaara R, Toro T, Bodmer W, Olschwang S, Olsen AS, Stratton MR, de la Chapelle A, Aaltonen LA (1998) A serine/threonine kinase gene defective in Peutz-Jeghers syndrome. Nature 391: 184-187.

Jemal A, Siegel R, Xu J, Ward E (2010) Cancer statistics, 2010. CA Cancer J Clin 60: 277-300.
Linehan WM, Srinivasan R, Schmidt LS (2010) The genetic basis of kidney cancer: a metabolic disease. Nat Rev Urol 7: 277-285.

Livak KJ, Schmittgen TD (2001) Analysis of relative gene expression data using real-time quantitative PCR and the $2(-$ Delta Delta C(T)) Method. Methods 25: 402-408.

Lizcano JM, Goransson O, Toth R, Deak M, Morrice NA, Boudeau J, Hawley SA, Udd L, Makela TP, Hardie DG, Alessi DR (2004) LKB1 is a master kinase that activates 13 kinases of the AMPK subfamily, including MARK/ PAR-1. EMBO J 23: 833-843.

Lu JP, Hou ZF, Duivenvoorden WC, Whelan K, Honig A, Pinthus JH (2012) Adiponectin inhibits oxidative stress in human prostate carcinoma cells. Prostate Cancer Prostatic Dis 15: 28-35.

Pantuck AJ, Zisman A, Belldegrun A (2001) Biology of renal cell carcinoma: changing concepts in classification and staging. Semin Urol Oncol 19: 72-79.

Rini BI, Campbell SC, Escudier B (2009) Renal cell carcinoma. Lancet 373: $1119-1132$.

Rini BI, Small EJ (2005) Biology and clinical development of vascular endothelial growth factor-targeted therapy in renal cell carcinoma. J Clin Oncol 23: 1028-1043.

Robb VA, Karbowniczek M, Klein-Szanto AJ, Henske EP (2007) Activation of the mTOR signaling pathway in renal clear cell carcinoma. J Urol 177: 346-352.

Sahin F, Maitra A, Argani P, Sato N, Maehara N, Montgomery E, Goggins M, Hruban RH, Su GH (2003) Loss of Stk11/Lkb1 expression in pancreatic and biliary neoplasms. Mod Pathol 16: 686-691.

Shackelford DB, Vasquez DS, Corbeil J, Wu S, Leblanc M, Wu CL, Vera DR, Shaw RJ (2009) mTOR and HIF-1alpha-mediated tumor metabolism in an LKB1 mouse model of Peutz-Jeghers syndrome. Proc Natl Acad Sci USA 106: $11137-11142$.

Shinojima T, Oya M, Takayanagi A, Mizuno R, Shimizu N, Murai M (2007) Renal cancer cells lacking hypoxia inducible factor (HIF)-1alpha expression maintain vascular endothelial growth factor expression through HIF-2alpha. Carcinogenesis 28: 529-536.

Sloan KA, Marquez HA, Li J, Cao Y, Hinds A, O’Hara CJ, Kathuria S, Ramirez MI, Williams MC, Kathuria H (2009) Increased PEA3/E1AF and decreased Net/Elk-3, both ETS proteins, characterize human NSCLC progression and regulate caveolin-1 transcription in Calu-1 and NCI-H23 NSCLC cell lines. Carcinogenesis 30: 1433-1442.

Sowter HM, Raval RR, Moore JW, Ratcliffe PJ, Harris AL (2003) Predominant role of hypoxia-inducible transcription factor (Hif)-1alpha versus Hif2alpha in regulation of the transcriptional response to hypoxia. Cancer Res 63: 6130-6134.

Taliaferro-Smith L, Nagalingam A, Zhong D, Zhou W, Saxena NK, Sharma D (2009) LKB1 is required for adiponectin-mediated modulation of AMPKS6K axis and inhibition of migration and invasion of breast cancer cells. Oncogene 28: 2621-2633.

Tong WH, Sourbier C, Kovtunovych G, Jeong SY, Vira M, Ghosh M, Romero VV, Sougrat R, Vaulont S, Viollet B, Kim YS, Lee S, Trepel J, Srinivasan R, Bratslavsky G, Yang Y, Linehan WM, Rouault TA (2011) The glycolytic shift in fumarate-hydratase-deficient kidney cancer lowers AMPK levels, increases anabolic propensities and lowers cellular iron levels. Cancer Cell 20: $315-327$.

Trojan J, Brieger A, Raedle J, Esteller M, Zeuzem S (2000) 5'-CpG island methylation of the LKB1/STK11 promoter and allelic loss at chromosome 19p13.3 in sporadic colorectal cancer. Gut 47: 272-276.

Viollet B, Horman S, Leclerc J, Lantier L, Foretz M, Billaud M, Giri S, Andreelli F (2010) AMPK inhibition in health and disease. Crit Rev Biochem Mol Biol 45: 276-295.

Wang X, Spandidos A, Wang H, Seed B (2012) PrimerBank: a PCR primer database for quantitative gene expression analysis, 2012 update. Nucleic Acids Res 40: D1144-D1149.

Zhuang ZG, Di GH, Shen ZZ, Ding J, Shao ZM (2006) Enhanced expression of LKB1 in breast cancer cells attenuates angiogenesis, invasion, and metastatic potential. Mol Cancer Res 4: 843-849.

This work is published under the standard license to publish agreement. After 12 months the work will become freely available and the license terms will switch to a Creative Commons AttributionNonCommercial-Share Alike 3.0 Unported License. 